\title{
Thousand words about alcohol use disorder in inflammatory bowel disease
}

\section{Dominika Wietrzykowska}

Department of Gastroenterology and Hepatology, University Hospital in Wrocław, Poland

(iD) https://orcid.org/0000-0001-5368-327X

Corresponding author: wietrzykowska_dominika@onet.pl

\section{Katarzyna Neubauer}

Department of Gastroenterology and Hepatology,

Wrocław Medical University, Poland

D https://orcid.org/0000-0003-3650-9311

DOI: https://doi.org/10.20883/medical.e506

\begin{abstract}
Keywords: ulcerative colitis, primary sclerosing cholangitis, colorectal cancer, liver transplantation, alcohol use disorders
\end{abstract}

Published: 2021-03-30

\begin{abstract}
How to Cite: Wietrzykowska D, Neubauer K. Thousand words about alcohol use disorder in inflammatory bowel disease. JMS [Internet]. 2021 Mar 30;90(1):e506. doi:10.20883/ medical.e506
\end{abstract}

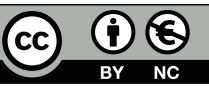

(C) 2021 by the author(s). This is an open access article distributed under the terms and conditions of the Creative Commons Attribution (CC BY-NC) licencse. Published by Poznan University of Medical Sciences

\begin{abstract}
Patients with inflammatory bowel disease (IBD), especially those with severe disease and extraintestinal manifestations, are more frequently affected by anxiety and depressive disorders compared to the healthy population. This in turn may favour the expansion of alcohol use disorders but the role of alcohol consumption in the development of IBD and its impact on IBD course remains controversial. Importantly, ethanol is a significant factor contributing to liver failure and increased risk of various malignancies, including colorectal cancer (CRC). Primary sclerosing cholangitis (PSC) is a fatal extraintestinal manifestation of IBD leading to liver failure and promoting the development of cholangiocarcinoma and colorectal cancer. Indeed, alcohol abuse by patients with IBD and PSC may promote the progression of those complications but is difficult to diagnose. The underlying disease may cause similar abnormalities in laboratory and imaging tests to ethanol thus masking the problem, therefore gastroenterologists should pay special attention to the alcohol consumption of IBD patients.
\end{abstract}

\section{Introduction}

Inflammatory bowel disease (IBD) is a chronic gastrointestinal disease encompassing ulcerative colitis (UC) and Crohn's disease (CD). The complex pathogenesis of IBD remains unclear, however, according to the conception of the "IBDactome", diseases arise from the interplay between genetic, immunological, microbiological, and environmental factors [1]. IBD is characterised by a wide spectrum of extraintestinal manifestations
(EMs), which may significantly impact the disease outcome. For instance, primary sclerosing cholangitis (PSC) is a fatal EM related to a higher risk of colorectal cancer (CRC) and cholangiocarcinoma. Furthermore, this chronic cholestatic liver disease may lead to liver failure, with more than $50 \%$ of individuals with PSC after 10-15 years of disease duration requiring liver transplantation (LTx) [2]. 


\section{Case report}

We report the case of a 26-year-old male patient with long-standing UC and PSC, hypothyroidism, depression, and nicotine addiction, repeatedly hospitalised in the Department of Gastroenterology and Hepatology due to disease flares or for monitoring of its course. The patient was admitted because of another endoscopic retrograde cholangiopancreatography to exchange the bile duct stents and start LTX qualification. During hospitalisation, laboratory tests showed signs of cholestasis (slightly elevated bilirubin, GGTP level over 50x above the upper limit of normal, alkaline phosphatase level close to 10x above the upper limit of normal), as well as elevated levels of aspartate and alanine aminotransferases. An extremely high level of thyrotropin was also alarming (over 20x exceeded the upper limit of the norm). The patient admitted the lack of regularity in the use of levothyroxine. Consequently, because of a previously diagnosed depressive disorder and in connection with qualification for LTx, a psychiatric consultation was performed that identified the harmful use of alcohol. The patient, despite previous numerous denials of alcohol consumption, admitted that he drinks mainly socially, consuming 6-8 beers a week with stronger drinks at the weekend despite knowing about the harmful effects of alcohol on the liver and is aware that he should stop. The psychiatrist recommended drug withdrawal psychotherapy for the patient. Further transplant qualification was postponed for the required abstinence period.

\section{Discussion}

The harmful use of alcohol is a drinking pattern that causes physical and mental damage, as well as psychological and social harm without being addicted to alcohol $[3,4]$. According to the World Health Organization (WHO, 2016), Polish men annually consume 23.8 litres of pure alcohol and Polish women consume 8.3 litres, with approximately $12.8 \%$ of the Polish population, predominantly men, abusing alcohol [5]. The overwhelming majority of these people do not meet the criteria for addiction and only use alcohol in a harmful way. It is worth noting that more than 3 million people worldwide die from the harmful use of alcohol each year [6].
Alcohol has been frequently considered as a potential risk factor for UC because it can directly damage the intestinal mucosa, modify the gut microbiome, increase bacterial translocation and interfere with digestion and nutrient absorption [7]. However, in an EPIC cohort study in 2017 of 262,451 people from six countries, no association was found between long-term alcohol consumption and IBD risk [8]. In a meta-analysis of sixteen studies involving 3689 cases, of which nine studies assessed the relation between alcohol consumption and UC risk, there was no significant association between alcohol consumption and the risk of developing UC [9]. Nevertheless, some clinical studies suggest that alcohol consumption may exacerbate the disease in patients already diagnosed with UC, possibly due to the effects of alcohol on the immune system by increasing gut permeability and antigen exposure or to the high sugar content of alcoholic beverages and associated osmotic diarrhoea [10, 11]. Furthermore, an EPIC study demonstrated that alcohol consumption predisposes to the development of cancer including CRC. Some epidemiological studies suggest that even moderate drinking increases the risk of CRC, which is the third most frequently diagnosed cancer in both men and women globally. Alcohol not only causes toxic effects through carcinogenic metabolites such as acetaldehyde but alcoholics themselves are predisposed to a poor diet and disturbances of the circadian rhythm, which may further intensify carcinogenesis [12].

The risk of developing CRC may reach $30 \% 20$ years after the diagnosis of concomitant IBD and PSC, therefore, alcohol abuse by patients with IBD and PSC significantly worsens the prognosis $[13,14]$. However, a 2012 study conducted on 96 patients with PSC by the Karolinska Institute in Sweden showed that only a small percentage of PSC patients consume excessive amounts of alcohol, with patients with significant liver fibrosis reducing their alcohol consumption after PSC diagnosis [15]. However, it should be noted that despite a diagnosis of liver disease, patients may additionally abuse alcohol so the underlying disease will cause similar changes in laboratory and imaging tests as the consumption of ethanol, thus masking the patient's alcohol problem. The problem of alcohol abuse by PSC patients is of particular concern as most of them require liver transplants over time. An observational study 
from France including 441 adult liver transplant recipients from 1991-2007 who survived > 6 months found that excessive drinking after LTX, regardless of the transplant reason, is associated with increased mortality [16]. Moreover, drinking alcohol after LTx is associated with an increased risk of graft rejection [17].

An additional argument for the active search for alcohol abuse is the fact that anxiety and depressive disorders are more common among IBD patients than in healthy individuals, and that they increase the risk of addiction to psychoactive substances including alcohol. In a study of 422 IBD patients at the Saarbrücken Clinic in Germany (2011), it was found that IBD patients with moderate/severe disease activity had higher rates of depression and anxiety compared to those with mild disease activity and age- and gender-matched healthy cohort. Additionally, the female gender was associated with an increased risk of anxiety in both IBD patients and the general population [18]. Inflammation, changes in the gut microbiota, and drug side effects influence the mental state of IBD patients but the relationship between mental disorders and IBD is not fully understood [19]. Many people with anxiety and depressive disorders tend to consume alcohol and other drugs, therefore it is important to pay special attention to drugs used by patients with IBD to provide them, if necessary, with timely psychological care to prevent addiction.

\section{Summary}

Patients suffering from UC and PSC are more predisposed to developing CRC than the general population, as well as to anxiety and depressive disorders, thereby the abuse of addictive substances. Alcohol can not only exacerbate IBD but more importantly, it also increases the risk of CRC, and like PSC, alcohol causes liver failure that often leads to LTx. Therefore, it is important that UC and PSC patients stop drinking alcohol and that gastroenterologists perform active screening for alcohol use disorders.

\section{Acknowledgements}

\section{Conflict of interest statement}

The authors declare no conflict of interest.

\section{Funding sources}

There are no sources of funding to declare.

\section{References}

1. de Souza HSP, Fiocchi C, lliopoulos D. The IBD interactome: an integrated view of aetiology, pathogenesis and therapy. Nature Reviews Gastroenterology \& Hepatology. 2017 Aug 23;14(12):739-749. https://doi. org/10.1038/nrgastro.2017.110

2. Emek E, Serin A, Sahin T, Yazici $P$, Yuzer $Y$, Tokat $Y$, Bozkurt B. Experience in Liver Transplantation Due to Primary Sclerosing Cholangitis: A Single Center Experience. Transplantation Proceedings. 2019 Sep;51(7):2439-2441.https://doi.org/10.1016/j.transproceed.2019.01.156

3. Alcohol. World Health Organization. https://www. who.int/health-topics/alcohol\#tab=tab_1

4. Alcohol-Use Disorders: Diagnosis, Assessment and Management of Harmful Drinking and Alcohol Dependence. National Collaborating Centre for Mental Health (UK). Leicester (UK): British Psychological Society; 2011.

5. Hammer J, Parent M, Spiker D. Global status report on alcohol and health 201865.1 (2018).. Global status report on alcohol. 2018;65(1).

6. World Health Organization. Alcohol. https://www. who.int/news-room/fact-sheets/detail/alcohol. Accessed 2018.

7. Mantzouranis G. Alcohol and narcotics use in inflammatory bowel disease. Annals of Gastroenterology. 2018; . https://doi.org/10.20524/aog.2018.0302

8. Bergmann MM, Hernandez V, Bernigau W, Boeing $\mathrm{H}$, Chan SSM, Luben R, Khaw K, van Schaik F, Oldenburg $B$, Bueno-de-Mesquita B, Overvad K, Palli D, Masala G, Carbonnel F, Boutron-Ruault M, Olsen A, Tjonneland A, Kaaks R, Katzke V, Riboli E, Hart AR. No association of alcohol use and the risk of ulcerative colitis or Crohn's disease: data from a European Prospective cohort study (EPIC). European Journal of Clinical Nutrition. 2017 Jan 25;71(4):512-518. https://doi. org/10.1038/ejcn.2016.271

9. Nie J, Zhao Q. Beverage consumption and risk of ulcerative colitis. Medicine. 2017 Dec;96(49):e9070. https://doi.org/10.1097/md.0000000000009070

10. Cohen $A B$, Lee $D$, Long MD, Kappelman MD, Martin CF, Sandler RS, Lewis JD. Dietary Patterns and Self-Reported Associations of Diet with Symptoms of Inflammatory Bowel Disease. Digestive Diseases and Sciences. 2012 Aug 26;58(5):1322-1328. https:// doi.org/10.1007/s10620-012-2373-3

11. Swanson GR, Sedghi S, Farhadi A, Keshavarzian A. Pattern of alcohol consumption and its effect on gastrointestinal symptoms in inflammatory bowel disease. Alcohol. 2010 May;44(3):223-228. https:// doi.org/10.1016/j.alcohol.2009.10.019

12. Rossi M, Anwar MJ, Usman A, Keshavarzian A, Bishehsari F. Colorectal Cancer and Alcohol Consumption-Populations to Molecules. Cancers. 2018 Jan 30;10(2):38. https://doi.org/10.3390/cancers 10020038

13. Wang R, Leong R. Primary sclerosing cholangitis as an independent risk factor for colorectal cancer in 
the context of inflammatory bowel disease: a review of the literature. World J Gastroenterol. $2014 \mathrm{Jul}$ 21;20(27):8783-9. https://doi.org/10.3748/wjg.v20. i27.8783 PMID 25083052

14. Hagström H. Alcohol Consumption in Concomitant Liver Disease: How Much is Too Much?. Current Hepatology Reports. 2017 Apr 22;16(2):152-157. https://doi.org/10.1007/s11901-017-0343-0

15. Hagström $\mathrm{H}$. Alcohol consumption in patients with primary sclerosing cholangitis. World Journal of Gastroenterology. 2012;18(24):3105. https://doi. org/10.3748/wjg.v18.i24.3105

16. Faure S, Herrero A, Jung B, Duny Y, Daures J, Mura $T$, Assenat E, Bismuth M, Bouyabrine $H$, DonnadieuRigole H, Navarro F, Jaber S, Larrey D, Pageaux G. Excessive alcohol consumption after liver transplantation impacts on long-term survival, whatev- er the primary indication. Journal of Hepatology. 2012 Aug;57(2):306-312. https://doi.org/10.1016/j. jhep.2012.03.014

17. Rice JP, Eickhoff J, Agni R, Ghufran A, Brahmbhatt R, Lucey MR. Abusive drinking after liver transplantation is associated with allograft loss and advanced allograft fibrosis. Liver Transplantation. 2013 Nov 27;19(12):1377-1386. https://doi.org/10.1002/lt.23762

18. Häuser W, Janke K, Klump B, Hinz A. Anxiety and depression in patients with inflammatory bowel disease. Inflammatory Bowel Diseases. 2011 Feb;17(2):621-632. https://doi.org/10.1002/ibd.21346

19. Kuźnicki P, Kempiński R, Neubauer K. The emerging role of mood disorders in inflammatory bowel diseases. Advances in Clinical and Experimental Medicine. 2020 Dec 31;29(12):1505-1510. https://doi. org/10.17219/acem/127676 